\title{
INTELIGENCIA EMOCIONAL, AUTOESTIMA Y PREVENCIÓN DE CONDUCTAS DE RIESGO DE LOS ADOLESCENTES
}

\author{
Luz Marina Ticona Pajares'; Segundo Cancino Morales ${ }^{t}$
}

\section{RESUMEN}

En una muestra de 114 adolescentes de la población accesible al Centro de Atención Integral del Adolescente, del CLAS Centro de Salud Leoncio Prado, se estudió si la inteligencia emocional y la autoestima son factores protectores en la prevención de conductas de riesgo de los adolescentes. Los resultados reflejan niveles de moderado a bajo en el mayor porcentaje de adolescentes de la muestra, por lo tanto, las dos variables no constituyen factores protectores.

Así mismo se encontró que, en los tres grupos estudiados, el mayor porcentaje de adolescentes tendría como factor de riesgo importante el ámbito familiar.

\section{A B STRACT}

In a 114 - teenagers - sample from an accessible population in the Adolescent Integral Attention Centrer (CLAS Centro de Salud Leoncio Prado) it was studied whether the emotional intelligence and self - esteem are protective factors to prevent risking behaviours in adolescents or not. The results show from moderate levels to a low level in the highest percentage of adolescent from the sample, 50 both variables are not protective factors.

In the same way, it was discovered that in the threse three studied groups, the highest percentage of adolescents would have the family environment.

\section{INTRODUCCIÓN}

La transición de la niñez a la vida adulta abarca aproximadamente la segunda década de vida de un individuo y se la reconoce como la etapa de la adolescencia que como proceso, lento, implica la transformación progresiva del niño dependiente en adulto independiente. Durante la adolescencia, el joven o la joven debe lograr consolidar su desarrollo somático, personal, emocional, intelectual y social. Este proceso se torna cada vez más difícil y complejo en una sociedad como la actual, signada por el avance vertiginoso del conocimiento, el fenómeno de la globalización y la pérdida de la individualidad. El éxito con el que transcurra la adolescencia de una persona dependerá del cúmulo de factores protectores con que cuente: inteligencia emocional, autoestima, proyecto de vida, etc. Estos le permitirán mantener estilos de vida saludables, prevenir las conductas de riesgo, responder adecuadamente a una sociedad que exige cada vez más una preparación más compleja.

Frente a la situación expuesta, y dada la urgente necesidad de brindar servicios de atención integral al adolescente con calidad y calidez, se plantea la interrogante siguiente: 
¿La inteligencia emocional y la autoestima son factores protectores en la prevención de conductas de riesgo de los adolescentes que atiende el Centro de Atención Integral del Adolescente, CLAS Leoncio Prado?

Si tomamos como referencia la Teoria de la unidad biosicosocial cultural y ecológica y la Teoria de sistemas, tenemos que considerar al adolescente como un todo cuyas partes se encuentran en interacción dinámica, formando una compleja organización; es decir: un todo que funciona como un sistema abierto, en el que los principales macrosistemas, relacionados con el bienestar y el desarrollo de los adolescentes, son: el biológico, el psicológico el social y el ecológico. Al interior de cada uno de estos -y entre ellos-, se producen una serie de interrelaciones dinámicas, no necesariamente armónicas en cuanto a su intensidad y velocidad. Un cambio favorable o desfavorable en uno de los elementos de un sistema puede alterar otros elementos de este o de otro sistema. Finalmente, estas teorias, además de permitir identificar las interrelaciones de los sistemas y sus componentes, permiten establecer los ejes de acción o intervención. (Marriner 1995, OPS/OMS, Serrano 1995 )

Por otro lado, la Teoria del enfoque de riesgo, que surgió de la asociación del concepto de vulnerabilidad con la noción de factor de riesgo, y que se entiende como una característica o circunstancia cuya presencia aumenta la probabilidad de que se produzca un daño o un resultado no deseado. Más aún: esta teoría se enriqueció con la incorporación del concepto de factor protector que permite utilizarla como un mecanismo no sólo para prevenir daños sino también para promover la salud cuando se actúa con anticipación. En consecuencia, la Teoría riesgo contribuye a determinar aquellas condiciones de naturaleza biológica, sicológica, social, cultural o ecológica que constituyen o anuncian la inminencia de un peligro o daño (factores de riesgo), y aumentan la probabilidad de consecuencias indeseables para el bienestar o desarrollo del adolescente; pero, a su vez, reconoce aquella característica bionatural, sicológica, social, cultural o ambiental, presente en un adolescente o un grupo, que favorece o actúa positivamente previniendo un daño, o potenciando favorablemente la condición de bienestar y desarrollo(factor protector).

Por una parte, puede considerarse que los factores protectores pueden radicar en los mismos adolescentes, en su ámbito familiar, en los grupos de pares, clubes deportivos, artísticos, en la comunidad representada por sus organizaciones sociales (de salud, de educación, de recreación, de justicia...). Y, por otra, la existencia de factores protectores aislados puede no tener un efecto visible. Los factores protectores son susceptibles de fortalecimiento y también pueden debilitarse y aun desaparecer

Además es pertinente mencionar el concepto de Resilencia entendido como la capacidad de reacción que pueden desarrollar los adolescentes cuando están expuestos a situaciones difíciles o agresiones. Se sabe que hay factores individuales, familiares y ambientales que actúan como tales fortaleciendo a las personas expuestas a una situación adversa, tanto en su aspecto físico, psicológico, social como ambiental. Algunos de estos factores, en el ámbito individual, son la autoestima, la inteligencia emocional, la autonomía, la educación, el proyecto de vida, la creatividad; en el ámbito familiar, el hogar estructurado, la tutoría, la recreación; en el ámbito comunitario, el ambiente de aceptación del adolescente y de respeto a sus derechos, etc.

Dentro de este marco de referencia, se ha creido conveniente estudiar dos de los factores protectores: la inteligencia emocional y la autoestima. Esto porque es evidente que la mayor parte de problemas en el proceso de desarrollo de los adolescentes están asociados a la falta de solidez en el desarrollo de factores protectores, lo que hace que los factores de riesgo encuentren un campo propicio y desarrollen conductas inapropiadas: embarazo precoz, accidentes, alcoholismo, drogadicción, etc.

Ser emocionalmente inteligente significa conocer las emociones propias y ajenas, su magnitud y sus causas. Poseer habilidades emocionales implica saber manejar las emociones a partir del conocimiento de las mismas y ser capaz de conducirlas de una manera tal que se llegue a mejorar el desarrollo personal y la calidad de vida. La inteligencia emocional fomenta las relaciones, crea posibilidades afectivas entre las personas, hace más cooperativo el trabajo y facilita el sentimiento de comunidad.

La autoestima es la valoración que tenemos de nosotros mismos, la opinión y el sentimiento que cada uno tiene acerca de sí, de los propios actos, valores y conductas; es la actitud hacia uno mismo y como tal es la forma de pensar, amar, sentir y comportarse consigo mismo.(Alcántara, 1993). El adolescente con 
autoestima alta siente confianza en sí, tiene estabilidad emocional, es responsable, competente, independiente, creativo, comunicativo y tiene buenas relaciones sociales, lo que va a repercutir en su éxito personal.

\section{OBJETIVOS}

- Describir las características de la autoestima e inteligencia emocional de los adolescentes.

- Determinar el nivel de autoestima e inteligencia emocional y su influencia en la prevención de conductas de riesgo del adolescente.

\section{HIPÓTESIS}

El nivel de autoestima y desarrollo de la inteligencia emocional de los adolescentes que de alguna manera se relacionan con el del Centro de Atención integral del Adolescente (CAINPA) son factores protectores en la prevención de conductas de riesgo.

\section{MATERIAL Y MÉTODOS}

El grupo de estudio estuvo conformado por aproximadamente 480 adolescentes entre 12 y 19 años, población a la que el Centro de Atención Integral del Adolescente del CLAS - Leoncio Prado brinda atención.

Para definir el tamaño de muestra se han considerado las exigencias del $95 \%$ de confianza $(Z=1,96)$, una varianza máxima que asegure un tamaño de muestra suficientemente grande $(P Q=0,25)$ y un error de muestreo del $8 \%(d=0,08)$. Para seleccionar la muestra, se hizo uso del muestreo por conglomerados, dividiendo a la población en tres conglomerados porque presentaban caracteristicas más o menos diferentes. En cada uno de ellos, se eligió al azar simple un número de hombres y mujeres, de acuerdo con los siguientes criterios de inclusión:

- Edades comprendidas entre los 10 y 19 años.

- Adolescentes de ambos sexos.

- Que no presenten enfermedad evidente.

En cada conglomerado, se seleccionó la muestra en forma proporcional, mediante el método de azar simple entre los adolescentes que reunian los criterios de inciusión, tomando contacto con ellos en los centros educativos de la jurisdicción del Centro de
Atención Integral del Adolescente. De tal manera que la muestra quedó conformada por 114:

$\begin{array}{ll}\text { Grupo A } & 30 \\ \text { Grupo B } & 40 \\ \text { Grupo C } & 44\end{array}$

Los instrumentos que se utilizaron en la recolección de la información fueron:

- Escala de valoración de nivel de autoestima del adolescente, propuesta por Clark-Clemens, a la que, después de una muestra piloto, se le hizo ciertas adaptaciones, especialmente en el uso de la terminología para adaptarla más al lenguaje de los adolescentes de la zona. La escala consta de 20 items, con tres alternativas de respuestas valoradas con $1,2,3$, ordenadas en forma aleatoria de manera que no siempre la alternativa $A$ tiene el valor 3 . La suma máxima de la puntuación es de 60 que corresponde al $100 \%$. Los resultados permitieron determinar los siguientes niveles:

- Nivel de autoestima alto : 48 A 60 puntos

- Nivel de autoestima promedio : 34 a 47 puntos

- Nivel de autoestima bajo : $20 \mathrm{~A} 33$ puntos

- Escala de valoración de nivel de inteligencia emocional. Se empleó el cuestionario de Steiner que consta de 6 partes, cada una de ellas contiene 6 situaciones, enunciados o afirmaciones, que se consignan con las letras A, B, C, D, E, F, y tienen 3 alternativas de respuesta, Sí, No, No Sé.

- Las preguntas A indagan sobre el adormecimiento emocional. Un estado en el cual la persona no tiene conciencia de sus emociones, es decir, sus emociones no son accesibles a su conciencia. Los resultados se califican de la manera siguiente:

2 a 0 Perfil alto (no hay adormecimiento emocional)

3 a 4 Perfil medio (se logran percibir algunas emociones)

5 a 6 Perfil bajo (adormecimiento emocionnal)

- Las preguntas B se ocupan de los síntomas físicos. En este estado de conciencia emocional, se experimentan las sensaciones que acompañan a las emociones, pero no se tiene conciencia de las emociones mismas. Los resultados se califican de la manera siguiente: 
2 a 0 Perfil alto (hay conciencia de las emociones)

3 a 4 Perfil medio (se experimentan sensaciones físicas y se perciben algunas emociones)

5 a 6 Perfil bajo (se experimentan sensaciones físicas pero no hay conciencia de las emociones)

- Las preguntas $C$ se refieren a una experiencia prístina caótica. En este estado, la persona es consciente de sus emociones, pero las experimenta con un elevado nivel de energía que no logra comprender ni expresar en palabras; la persona es muy vulnerable y generalmente no controla sus emociones. Los resultados se califican de la manera siguiente:

2 a 0 Perfil bajo (hay conciencia de las emociones, pero no las comprende ni puede expresarlas.)

3 a 4 Perfil medio (tiene conciencia de las emociones, logra comprender algunas pero tiene dificultad para expresarlas.)

5 a 6 Perfil alto (tiene conciencia de las emociones, las comprende y puede expresarlas en palabras.)

- Las preguntas D indagan acerca de la diferenciación. Este nivel de conciencia revela el reconocimiento de las diferentes emociones, y de su respectiva intensidad y de la posibilidad de hablar a los demás acerca de ellas. Los resultados se califican de la manera siguiente:

2 a 0 Perfil bajo (reconoce las emociones, pero no la intensidad de las mismas, ni puede hablar de ellas con los demás)

3 a 4 Perfil medio (reconoce las emociones, diferencia generalmente la intensidad, tiene dificultad para hablar de ellas con los demás.)

5 a 6 Perfil alto (reconoce las emociones, y diferencia la intensidad de la mismas, habla acerca de ellas con los demás)
- Las preguntas E se refieren a la empatía. Es decir, la posibilidad que se tiene de percibir las emociones de otras personas. Los resultados se califican de la manera siguiente:

2 a 0 Perfil bajo (generalmente no percibe las emociones de los demás.)

3 a 4 Perfil medio (tienen dificultades para percibir las emociones de los demás.)

5 a 6 Perfil alto (percibe bien las emociones de los demás)

- Las preguntas F indagan sobre la interactividad. Es decir, sobre el nivel más sofisticado de la conciencia, ya que no sólo requiere que uno sepa lo que uno mismo siente y lo que siente el otro, sino que, además, hace falta que pueda anticipar cómo dos personas diferentes van a reaccionar en una situación dada. Los resultados se califican de la manera siguiente:

2 a 0 Perfil bajo (generalmente no percibe sus emociones ni las de los demás.)

3 a 4 Perfil medio (tienen dificultades para percibir sus emociones y las de los demás, no sabe cómo pueden reaccionar frente a determinadas situaciones)

5 a 6 Perfil alto (percibe bien sus emociones y la de los demás, puede anticipar la reacción de las personas)

- Escala de valoración de conductas de riesgo: En este caso se tomó como referencia el instrumento diseñado por América Medical Association,(1997). Las experiencias de aplicación en el Centro de Atención Integral del Adolescente Centro de Salud Leoncio Prado permitieron modificar este cuestionario reduciéndolo a 8 subvariables y 31 items Se establece el riesgo de la siguiente manera:

- Alto riesgo : de 4 a 5 puntos

- Mediano riesgo de 2 a 3 puntos

- Bajo riesgo de 1 a 0 puntos 


\section{RESULTADOS}

\section{Variable Autoestima}

Cuadro 1. Nivel de autoestima de los adolescentes segin grupos de estudio y sexo. Tacna 2000.

\begin{tabular}{|c|c|c|c|c|c|c|c|c|c|c|c|c|c|c|}
\hline \multirow{3}{*}{$\begin{array}{l}\text { GRUPOS } \\
\text { NN.AUTE. }\end{array}$} & \multicolumn{4}{|c|}{ BAJA } & \multicolumn{4}{|c|}{ MEDIA } & \multicolumn{4}{|c|}{ ALTA } & \multirow{2}{*}{\multicolumn{2}{|c|}{ Total }} \\
\hline & Masculi. & Femeni. & Masculi. & Femeni. & Masculi. & Femeni. & Masculi. & Femeni. & Masculi. & Femeni. & Masculi. & Femeni. & & \\
\hline & $\mathrm{N}^{\circ}$ & $\%$ & $\mathrm{~N}^{\circ}$ & $\%$ & $\mathrm{~N}^{\circ}$ & $\%$ & $\mathrm{~N}^{\circ}$ & $\%$ & $N^{0}$ & $\%$ & $\mathrm{~N}^{0}$ & $\%$ & $\mathrm{~N}^{\circ}$ & $\%$ \\
\hline Grupo A & & & & & 16 & 53.33 & 10 & 33.34 & 1 & 3.33 & 3 & 10.00 & 30 & 100.00 \\
\hline Grupo B & 1 & 2.50 & 1 & 2.50 & 16 & 40.00 & 21 & 52.50 & & & 1 & 2.50 & 40 & 100.00 \\
\hline Grupo C & & & & & 26 & 59.10 & 5 & 11.36 & 12 & 27.27 & 1 & 2.27 & 44 & 100.00 \\
\hline TOTAL & 1 & 0.88 & 1 & 0.88 & 58 & 50.88 & 36 & 31.58 & 13 & 11.40 & 5 & 4.38 & 114 & 100.00 \\
\hline
\end{tabular}

Los datos sobre el nivel de autoestima de los adolescentes de la muestra indican que en los tres grupos (ver Cuadro 1), tanto hombres como mujeres tienen predominantemente un nivel medio de autoestima; sin embargo, se observa una figera diferencia en el grupo $\mathrm{C}$, es el único donde se percibe un $27,27 \%$ de varones ubicados en el nivel de autoestima alto. Estos resultados difieren con los obtenidos en otros estudios en los que mayores porcentajes se ubican en el nivel alto. Considerando to afirmado por Clark, Clemens y Bean (1996) en relación con el nivel medio de autoestima, que es el que predomina en los adolescentes observados, se puede inferir que estos se sienten inseguros, incapaces de hacer cosas; lo que podría, en el futuro, llevarlos a cerrarse frente a las posibilidades de éxito. Por lo tanto, este nivel de autoestima se convierte en un factor de riesgo.

\section{Variable inteligencia emocional:}

Figura 1. Promedios de las caracteristicas de la conciencia emocional en los grupos de adolescentes estudiados.

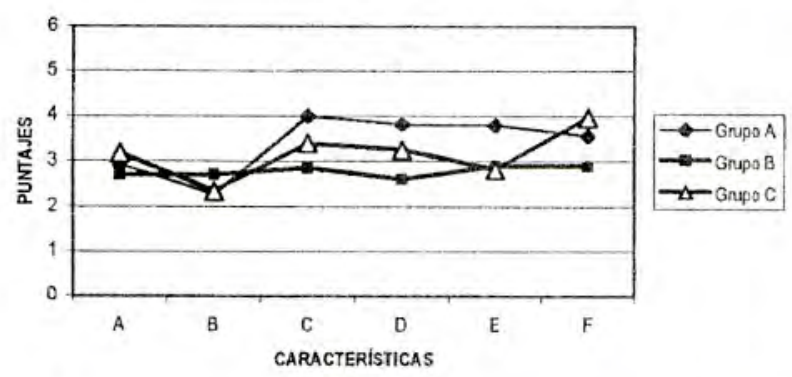

La inteligencia emocional es considerada como un factor protector en tanto que permite comprender las emociones, expresarlas de una manera productiva, desarrollar la capacidad para escuchar a los demás y sentir empatía respecto de sus emociones. Al respecto, el cuestionario de Steiner ha permitido determinar los siguientes niveles en el desarrollo de conciencia emocional en la muestra de estudio:

- Adormecimiento emocional (datos de la columna A): como se observa en la figura 1 , los puntajes promedios alcanzados por los grupos $A, B, C,(2,93$, 2,70 y 3,18 ) indican que, en este aspecto de la conciencia emocional, tienen un nivel de desarrollo que los ubica dentro del grupo de personas que no ha prestado demasiada atención a sus sentimientos. Esto quiere decir que la mayor parte de las veces no tienen conciencia de lo que sienten; es decir, las emociones no son parte de su vida normal. Ello no les permite un manejo adecuado de sus emociones; por lo que, como es previsible, no logran desarrollar sentimientos de empatía y habilidades de interacción.

Los porcentajes, que se ubican en los puntajes 2, 1 y 0 (grupo A 33, 33\%, $16,67 \%$, grupo B $32,50 \% 10,00 \%$ y $7,50 \%$; grupo $C 11,36,13,64$ y $6,82 \%$ ), demuestran que los adolescentes han logrado desarrollar suficientemente este aspecto de la conciencia emocional. Esto es: perciben sus emociones, por to que están en mejor situación de continuar el proceso de desarrollo de la inteligencia emocional.

Por otro lado, los adolescentes que alcanzan puntajes 5,6 (Grupo A 23,33\%, grupo B 12,50\% y grupo C 22,73 y $2,27 \%$ ) permiten observar que se encuentran en mayor riesgo, porque estos puntajes revelan un adormecimiento emocional. 
- Síntomas físicos (datos de la columna B). Como se demuestra en la Figura $\mathrm{N}^{\circ} 1$, el puntaje promedio alcanzado por los grupos $A, B, C,(2,27,2,70$ y 2,34$)$ indica que los adolescentes tienen un nivel de desarrollo que los ubica dentro del grupo de personas que experimentan sensaciones fisicas que acompañan a las emociones, pero que no poseen conciencia clara de las mismas. En este caso, las personas suelen recurrir a los analgésicos u otras sustancias para disminuir los síntomas físicos que en realidad tienen origen emocional. En este estado, los conflictos no desaparecen, permanecen sin resolver y es probable que los adolescentes se lastimen a si mismos o lastimen a los demás y esto conlleva que se sientan culpables y disminuya aún más su conciencia emocional.

Los que se ubican en los puntajes 2,1 y 0 (grupo $A$ $30,00 \%, 16,67 \%$ y $6,67 \%$, grupo B $30,00 \%, 27,50 \%$ y $7,50 \%$, grupo C $29,55,18.18$ y $9,09 \%$ ) son los que experimentan algunos signos físicos que acompañan a las emociones. Estos de alguna manera estarían haciendo conciencia de sus emociones, lo que les permite manejarlas mejor $y$, por consiguiente, lograr mayores niveles de desarrollo.

Por otro lado, los adolescentes que alcanzan puntajes 5,6 (grupo A 6,67\%, grupo B 12,50\%, 2.50\% y grupo $C 6,82 \%$ ) son de los que se encuentran en mayor riesgo, en la medida que revelan una gran somatización de las emociones.

- Experiencia prístina caótica La Figura 1, columna $C$, permite examinar el puntaje promedio alcanzado por los grupos A, B, C, $(4,00,2,85$ y 3,39$)$, cifras que establecen diferencias entre los grupos y permiten inferir que los adolescentes que han logrado mayor conciencia de sus emociones son los de los grupos $\mathrm{A}$ y $C,(4,00,3,39)$, lo que se constituye en base importante para continuar el desarrollo de su conciencia emocional. El grupo B se caracteriza por tener aún muchas dificultades en tomar conciencia de sus emociones, situación que los pone en desventaja en su relación consigo mismo y con los demás.

Por otro lado, los que alcanzan puntajes 5,6 (grupo A $26,67 \%, 20,00 \%$, grupo B $15,00 \%$ y grupo C $18,18 \%$, $11,36 \%$ ) son los que tienen conciencia de sus emociones, pero que no siempre saben qué hacer con ellas; sin embargo, esta se constituye en buena base para lograr un buen manejo de sus emociones.

- Diferenciación Los datos de columna D (Figura 1) muestran el puntaje promedio alcanzado por los grupos
A, B, C, $(3,83,2,60$ y 3,25$)$, cifras que establecen diferencias entre los grupos. En este caso, han logrado mayor conciencia y diferenciación de la intensidad de sus emociones los grupos A y C, $(3,83,3,25)$, esto se constituye en una variable positiva para el logro de un alto nivel de desarrollo de su conciencia emocional. El grupo B se caracteriza por experimentar aún limitaciones para reconocer y diferenciar la intensidad de sus emociones

Los porcentajes de adolescentes, cuyos puntajes son 5,6 (grupo A 13,33\%, 13,33\%, grupo B 10,00\% y grupo C $15,91 \%, 19,09 \%)$, han logrado un buen nivel de diferenciación de las emociones, de su intensidad y predisposición para hablar de ellas, lo que da ventajas para lograr un manejo satisfactorio de estas.

- La empatía. Los datos de la columna E (Figura 1) presentan los puntajes promedios alcanzados por los grupos A, B, C, $(3,80,2,90$ y 2,82$)$ : se observan diferencias. El grupo $A(3,80)$ es el que logra percibir alguna de las emociones de las otras personas, de manera que puede interactuar mejor; mientras que los grupos $\mathrm{B}$ y C $(2,90$ y 2,82$)$ no perciben adecuadamente las emociones de los demás, lo que les dificulta sus relaciones interpersonales. Por otro lado, son relativamente bajos los porcentajes de los que alcanzan puntajes 5,6 (grupo A $13,33 \%, 6,67 \%$, grupo B $15,00 \%$ y grupo $C 6,82 \%, 4,55 \%$ ), esto sugiere que han logrado habilidad en la percepción de las emociones de otras personas $y$, en consecuencia, muestran un buen nivel de desarrollo emocional.

- La interactividad (datos de la columna F, Figura 1). Se observa que el puntaje promedio alcanzado por los grupos A, B, C, $(3,56,2,70$ y 3,95$)$, establece diferencias. Los grupos A y C $(3,80$ y 3,95$)$ son los que revelan que el desarrollo en este aspecto estaría en proceso. El grupo $B(2,70)$ muestra un nivel de desarrollo incipiente, esto dificulta fundamentalmente la relación con los demás ya que no se ha adquirido la habilidad de prever la forma cómo las otras personas pueden reaccionar ante diversas situaciones. Por otro lado, son relativamente bajos los porcentajes de adolescentes cuyos puntajes 5, 6 (grupo A 16,67, $.13,33 \%$, grupo B $7,50 \%, 7,50 \%$ y grupo C $9,09 \%$, $18,18 \%$ ) demuestran que son los que han logrado habilidad para percibir las emociones y anticipar la forma cómo las personas pueden reaccionar en diferentes situaciones. 


\section{Factores de riesgo}

Cuadro 2. Nivel de riesgo en 7 variables estudiadas en los adclescentes grupo A. Tacna 2000.

\begin{tabular}{|l|c|c|c|c|c|c|c|c|}
\hline \multirow{2}{*}{ Variables } & \multicolumn{5}{|c|}{ Niveles de Riesgo } & \multicolumn{2}{c|}{ Total } \\
\cline { 2 - 9 } & \multicolumn{2}{|c|}{ Bajo } & Moderado & \multicolumn{2}{c|}{ Alto } & \multicolumn{2}{c|}{} \\
\cline { 2 - 9 } & $N^{0}$ & $\%$ & $N^{\circ}$ & $\%$ & $N^{\circ}$ & $\%$ & $N^{\circ}$ & $\%$ \\
\hline Riesgo familiar & 5 & 16.67 & 20 & 66.67 & 5 & 16.67 & 30 & 100.00 \\
\hline Escuela & 22 & 73.33 & 8 & 26.67 & 0 & 0.00 & 30 & 100.00 \\
\hline Amistades & 18 & 60.00 & 5 & 16.67 & 7 & 23.33 & 30 & 100.00 \\
\hline $\begin{array}{l}\text { Violencia y } \\
\text { seguridad }\end{array}$ & 17 & 56.67 & 12 & 40.00 & 1 & 3.33 & 30 & 100.00 \\
\hline $\begin{array}{l}\text { Tabaco, alcohol y } \\
\text { otras drogas }\end{array}$ & 12 & 40.00 & 15 & 50.00 & 3 & 10.00 & 30 & 100.00 \\
\hline Emociones & 13 & 43.33 & 15 & 50.00 & 2 & 6.67 & 30 & 100.00 \\
\hline Desarrollo & 15 & 50.00 & 14 & 46.67 & 1 & 3.33 & 30 & 100.00 \\
\hline
\end{tabular}

Como se puede observar en el Cuadro $\mathrm{N}^{\circ} 2$, en el grupo A de la muestra, el $66,67 \%$ y el $16,67 \%$ alcanzan niveles de riesgo moderado y alto respectivamente, en el ámbito familiar, y en tabaco alcohol y otras drogas $(50,00,10 \%)$. Las demás variables estudiadas distribuyen sus porcentajes entre riesgo moderado y bajo.

Cuadro 3. Nivel de riesgo en 7 variables estudiadas en los adolescentes grupo B. Tacna 2000.

\begin{tabular}{|c|c|c|c|c|c|c|c|c|}
\hline \multirow{3}{*}{ Variables } & \multicolumn{6}{|c|}{ Niveles de Riesgo } & \multirow{2}{*}{\multicolumn{2}{|c|}{ Total }} \\
\hline & \multicolumn{2}{|c|}{ Bajo } & \multicolumn{2}{|c|}{ Moderado } & \multicolumn{2}{|c|}{ Alto } & & \\
\hline & $\mathrm{N}^{0}$ & $\%$ & $\mathrm{~N}^{\circ}$ & $\%$ & $\mathrm{~N}^{0}$ & $\%$ & $\mathrm{~N}^{\circ}$ & $\%$ \\
\hline Riesgo familiar & 9 & 22.50 & 26 & 65.00 & 5 & 12.50 & 40 & 100.00 \\
\hline Escuela & 29 & 72.50 & 9 & 22.50 & 2 & 5.00 & 40 & 100.00 \\
\hline Amistades & 14 & 35.00 & 16 & 40.00 & 10 & 25.00 & 40 & 100.00 \\
\hline $\begin{array}{l}\text { Violencia y } \\
\text { seguridad }\end{array}$ & 23 & 57.50 & 15 & 37.50 & 2 & 5.00 & 40 & 100.00 \\
\hline $\begin{array}{l}\text { Tabaco, alcohol y } \\
\text { otras drogas }\end{array}$ & 35 & 87.50 & 4 & 10.00 & 1 & 2.50 & 40 & 100.00 \\
\hline Emociones & 17 & 42.50 & 20 & 50.00 & 3 & 7.50 & 40 & 100.00 \\
\hline Desarrallo & 28 & 70.00 & 10 & 25.00 & 2 & 5.00 & 40 & 100.00 \\
\hline
\end{tabular}

Los datos del Cuadro 3 , grupo $B$, permiten observar que el $65,00 \%$ y el $12,50 \%$ de estos adolescentes alcanzan niveles de riesgo moderado y alto respectivamente, en el ámbito familiar, amistades $(40,00 \%, 25 \%)$. Las otras variables estudiadas distribuyen sus porcentajes entre riesgo moderado y bajo.

De los factores de riesgo estudiados, los que alcanzan mayores porcentajes entre riesgo moderado y alto son: riesgo familiar, violencia y seguridad, tabaco, alcohol y otras drogas. En cambio, los factores escuela, amistades, emociones, desarrollo, distribuyen sus mayores porcentajes entre el riesgo moderado y bajo.

Cuadro 4. Nivel de riesgo en 7 variables estudiadas en los adolescentes grupo C. Tacna 2000.

\begin{tabular}{|l|c|c|c|c|c|c|c|c|}
\hline \multirow{2}{*}{ Variables } & \multicolumn{5}{|c|}{ Niveles de Riesgo } & \multicolumn{2}{c|}{ Total } \\
\cline { 2 - 9 } & \multicolumn{2}{|c|}{ Bajo } & \multicolumn{2}{c|}{ Moderado } & \multicolumn{2}{c|}{ Alto } & \multicolumn{2}{c|}{} \\
\cline { 2 - 9 } & $\mathrm{N}^{\circ}$ & $\%$ & $\mathrm{~N}^{\circ}$ & $\%$ & $\mathrm{~N}^{\circ}$ & $\%$ & $\mathrm{~N}^{\circ}$ & $\%$ \\
\hline Riesgo familiar & 16 & 36.36 & 21 & 47.73 & 7 & 15.91 & 44 & 100.00 \\
\hline Escuela & 32 & 72.73 & 11 & 25.00 & 1 & 2.27 & 44 & 100.00 \\
\hline Amistades & 18 & 40.91 & 25 & 56.82 & 1 & 2.27 & 44 & 100.00 \\
\hline $\begin{array}{l}\text { Violencia y } \\
\text { seguridad }\end{array}$ & 13 & 29.55 & 22 & 50.00 & 9 & 20.45 & 44 & 100.00 \\
\hline $\begin{array}{l}\text { Tabaco, alcohol y } \\
\text { otras drogas }\end{array}$ & 18 & 40.91 & 19 & 43.18 & 7 & 15.91 & 44 & 100.00 \\
\hline Emociones & 26 & 59.09 & 16 & 36.36 & 2 & 4.55 & 44 & 100.00 \\
\hline Desarrollo & 20 & 45.45 & 19 & 43.18 & 5 & 11.36 & 44 & 100.00 \\
\hline
\end{tabular}

El Cuadro 4 muestra los datos recogidos en el grupo $\mathrm{C}$, donde el $47,73 \%$ y el $15,91 \%$ de estos adolescentes alcanzan niveles de riesgo moderado y alto respectivamente, en el ámbito familiar y violencia y seguridad $(50,00 \%, 20,45 \%)$. Las otras variables estudiadas distribuyen sus porcentajes entre riesgo moderado y bajo.

Finalmente, como se puede observar en los cuadros $\mathrm{N}^{\circ} 2,3,4$, de los factores de riesgo estudiados, el que alcanza mayores porcentajes entre riesgo moderado y alto en los tres grupos esiudiados, es el ámbito familiar. Los factores (grupo A) tabaco, alcohol y otras drogas (grupo B), amistades (grupo C) violencia y seguridad alcanzan estos mismos porcentajes en grupos diferentes. Esto sugiere de alguna manera, que el entorno que rodea a estos diferentes grupos de adolescentes juega un papel importante en los riesgos a que están expuestos. 


\section{CONCLUSIONES}

1. Los adolescentes tienen un nivel medio de autoestima (hombres 50,88 , mujeres $31,58 \%$ ) con leve tendencia hacia el nivel de autoestima alta (hombres, $11,40 \%$; mujeres $4.38 \%$ ).

2. Los grupos $A, B, C$, en los aspectos de adormecimiento emocional (A), síntomas físicos $(B)$ tienen puntajes promedios que van desde 2,27 al 3,18 , lo que indica que hay deficiencia en la percepción e identificación de las emociones y, en consecuencia, dificultad para alcanzar desarrollo satisfactorio de los otros aspectos de la inteligencia emocional.

3. Los grupos $A, B, C$, en los aspectos de Experiencia prístina caótica (C), diferenciación (D) logran puntajes promedios que van de 2,60 a 4,00 . Ubica a la muestra entre un perfil emocional medio y bajo; es decir, los adolescentes experimentan algunas emociones, tienen dificultad para diferenciar la intensidad y hablar de las mismas con otras personas.

4. Los grupos A, B, C, en los aspectos de empatía

$(E)$ e interactividad $(F)$, tienen puntajes promedios que van de 2,82 a 3,95 . Ubica a los grupos en un perfil de la conciencia emocional entre medio y bajo. Ello significa que los adolescentes perciben los sentimientos de los demás; pero, generalmente, no saben qué hacer con ellos, hecho que les dificulta su proceso de interacción con los demás.

5. Al individualizar los grupos de la muestra, el perfil de la inteligencia emocional del grupo $\mathrm{B}$, en todos los aspectos evaluados, mantiene puntajes promedios entre 2,60 y 2,90 , por lo que se ubica en un nivel bajo de desarrollo de la inteligencia emocional.

6. En la muestra de estudio, la autoestima y la inteligencia emocional no constituyen factores protectores potentes para los adolescentes.

7. Los factores que constituyen riesgo moderado y alto para los adolescentes son: familia, violencia y seguridad, tabaco, alcohol y otras drogas.

8. Los factores escuela, amistades, emociones, desarrollo, constituyen riesgo moderado y bajo.

MARTIN, D. y BOECK, k. (1997): Qué es inteligencia emocional. Editorial EDAF, S.A. Espana.

MONBOURQUETTE, Jean (1996): La comunicación familiar. Editorial Trillas, S.A. México.

SILVER, Tomás y OTROS (1992): Manual de medicina de la adolescencia. Editado por la Organización Panamericana de la Salud.

STEINER, Claude. y PERRY, Paul (1998): La educación emocional. Editorial Javier Vergara Editor.Argentina.

TORRES TASSO Y LUNA ANTEPARRA, J (1995): Desarrollo de la autoestima en niños $y$ adolescentes. Editado por Centro B f. Skinner. Lima. 